\title{
Gremlin-1 C-Terminus Regulates Function of Macrophage Migration Inhibitory Factor (MIF)
}

\author{
Sandra Beck ${ }^{\mathrm{a}}$ Thomas Simmet ${ }^{\mathrm{b}}$ Iris Müller ${ }^{\mathrm{a}}$ Florian Lang ${ }^{\mathrm{a}, \mathrm{c}}$ Meinrad Gawaz \\ aMedizinische Klinik III, Kardiologie und Kreislauferkrankungen, University of Tübingen, Tübingen, \\ bInstitute of Pharmacology of Natural Products and Clinical Pharmacology, Ulm University, Ulm, \\ 'Physiologisches Institut, University of Tübingen, Tübingen, Germany
}

\section{Key Words}

Gremlin-1 Macrophage migration inhibitory factor • Loss-of-function mutant • Monocytes • Migration - Differentiation

\begin{abstract}
Background/Aims: The counterbalance of macrophage migration inhibitory factor (MIF) and Gremlin-1 is a useful tool to predict the acuity of coronary artery disease (CAD) and plaque stability. Gremlin-1 is an endogenous antagonist of MIF and therefore influences plaque vulnerability. This study was designed to elucidate the mechanistic basis determining the biophysical binding of Gremlin-1 to MIF. Methods: An in silico model suggested that several charged C-terminal amino acids are crucial in mediating Gremlin-1/MIF-binding. We produced several single amino acid exchange mutants of Gremlin-1 by site-directed mutagenesis. These Gremlin-1 mutants were tested for their ability to reduce MIF effects on monocytes. Results: We observed that the critical element of the Gremlin-1 molecule for regulating MIF-induced chemotactic activity lies at the $\mathrm{C}$-terminal region. A single amino acid exchange of an arginine to an alanine residue is sufficient to abolish the antagonistic effect of Gremlin-1 on MIF. Therefore, the Gremlin-1 mutant R172A failed to reduce MIF-induced monocyte differentiation into macrophages. Conclusion: Gremlin-1 C-terminus is essential for antagonizing MIF effects. Our results could offer a novel strategy utilizing Gremlin-1 to target pro-inflammatory effects of MIF in various diseases.

\section{Introduction}

Gremlin-1 is a bone morphogenetic protein (BMP) antagonist and interacts with BMP2, BMP-4 and BMP-7 [1]. Gremlin-1 plays an important role in embryogenesis and organ development via inhibition of BMP-mediated signaling pathways, especially in lungs, kidneys, heart, and the bone formation of body axis and limbs [2, 3]. The fact that the constitutive Gremlin-1 knockout mouse is neonatally lethal emphasizes its essential role




\section{Cellular Physiology Cell Physiol Biochem 2016;38:801-808 \begin{tabular}{ll|l} 
DOI: 10.1159/000443035 & O 2016 The Author(s). Published by S. Karger AG, Basel \\
and Biocherger.com/cpb
\end{tabular} \\ Beck et al.: Gremlin-1 Mutants}

during embryogenesis [4]. Gremlin-1 and its homologs are highly conserved glycoproteins throughout different species and belong to the cysteine knot superfamily, together with proteins like TGF- $\beta$ and VEGF. Within this cysteine knot superfamily Gremlin-1 is classified as a member of DAN/Cerberus protein family and encodes a $23 \mathrm{kDa}$ non-glycosylated form, which can also appear as a $28 \mathrm{kDa}$ glycosylated form.

MIF (macrophage migration inhibitory factor) is a cytokine that has chemokine like functions. It directs the chemotaxis and recruitment of monocytes, their differentiation, substantiating their subsequent accumulation at atherosclerotic lesions [5, 6]. During the development of atherosclerotic plaques, monocyte recruitment and formation of macrophages are important events [7]. We showed previously that Gremlin-1 can act as an antagonist of MIF engaging in a high affinity binding with MIF as evidenced by surface plasmon resonance analysis [8]. We suggested the plasma Gremlin-1/MIF ratio as a predictive marker for classifying the severity of coronary artery disease (CAD) and to estimate plaque vulnerability [9].

\section{Materials and Methods}

\section{Generation of plasmids, PCR, primers}

The pcDNA5FRT-TO plasmid for expression of wild type Gremlin-1 was synthesized by GeneArt AG (Regensburg, Germany). Gremlin-1 mutant plasmids were obtained by a site-directed mutagenesis kit (Agilent Technologies Sales \& Services GmbH \& Co. KG, Waldbronn, Germany) using the wild type Gremlin-1 plasmid as template. Primer pairs for mutagenesis are listed below and were purchased from Eurofins Genomic GmbH (Ebersberg, Germany). Mutagenesis PCR was performed according to the manufacturer's manual. Numbering of the amino acid residues was chosen according to the full length human wild type Gremlin-1 protein (UniProt ID: 060565).

Primer namePrimer sequence $5^{\prime}$ '... 3'

K145A_fw GTC CTG CTC CTT CTG CGC GCC CAA GAA ATT CAC

K145A_rev GTG AAT TTC TTG GGC GCG CAG AAG GAG CAG GAC

K147A_fw GCT CCT TCT GCA AGC CCG CGA AAT TCA CTA CCA TG

K147A_rev CAT GGT AGT GAA TTT CGC GGG CTT GCA GAA GGA GC

R169A_fw CCC CAC CAA GAA AAA GGC CGT GAC CAG AGT GAA GCT TCA CTC T

R169A_rev CTT CAC TCT GGT CAC GGC CTT TTT CTT GGT GGG G

R172A_fw CAA GAA AAA GCG CGT GAC CGC TGT GAA GCA GTG CCG GTG CGC A

R172A_rev GCA CCG GCA CTG CTT CAC AGC GGT CAC GCG CTT TTT CTT G

\section{Cell isolation and cell lines}

Human monocytes were isolated from peripheral venous blood samples by adherence depletion following differential centrifugation through a Ficoll-Paque gradient as described previously [10]. Flp- $\mathrm{In}^{\mathrm{TM}}$ CHO cells were grown following the recommendations and instructions of the manufacturer (Invitrogen ${ }^{\mathrm{TM}}$, Life Technologies GmbH, Darmstadt, Germany).

\section{Protein production and purification}

Protein production was performed in Flp- $\mathrm{In}^{\mathrm{TM}}-\mathrm{CHO}$ cells after stable transfection as previously described [11-13]. As transfection reagent Lipofectamine2000 (Invitrogen $^{\mathrm{TM}}$, Life Technologies GmbH, Darmstadt, Germany) was used following the manufacturer's instructions. In brief, protein was produced by Flp- $\mathrm{In}^{\mathrm{TM}}$-CHO cells for one week. Protein purification was performed by ProteinA-Agarose precipitation (Pierce $^{\mathrm{TM}}$ Recombinant Protein A Agarose, Thermo Fisher Scientific, Life Technologies GmbH, Darmstadt, Germany). Protein concentrations were determined with anti-human IgG ELISA (Helvetica Healthcare, Switzerland) according to manufacturer's manual.

Monocyte chemotaxis

Migration of human monocytes was performed in RPMI1640 medium with stable glutamine supplemented with 10\% FCS (fetal calf serum) and 1\% penicillin/streptomycin $(100 \mu \mathrm{g} / \mathrm{ml}$ each) using 


\section{Cellular Physiology Cell Physiol Biochem 2016;38:801-808 \begin{tabular}{ll|l} 
DOI: 10.1159/000443035 & $\begin{array}{l}\text { O } 2016 \text { The Author(s). Published by S. Karger AG, Basel } \\
\text { www.karger.com/cpb }\end{array}$ \\
\cline { 2 - 3 }
\end{tabular}

a 48-well-modified Boyden chamber (Neuro Probe Inc., Gaithersburg, USA) as described previously [11] equipped with a $5 \mu \mathrm{m}$ polycarbonate filter membrane. In brief, the chemoattractant MIF (20 ng/ml) was preincubated with the wild type or mutant Gremlin-1 fusion protein, or control respectively, for 30 min at room temperature. Placing the MIF/Gremlin-1 mixture in the lower chamber, the monocytes were loaded onto the upper chamber and allowed to migrate at $37^{\circ} \mathrm{C}$ and $5 \% \mathrm{CO}_{2}$ for 4 hours. After migration the cells on the membrane filter were fixed in methanol and subsequently stained with May-Grünwald and Giemsa reagents.

In vitro model of macrophage and foam cell differentiation

Human monocytes were differentiated into macrophages/foam cells over 6 days. The effects of Gremlin-1 on formation of macrophages/foam cells were studied in vitro as described previously [14-16]. The formation of macrophages and foam cells was analyzed in the presence of MIF ( $20 \mathrm{ng} / \mathrm{ml}$ ) by morphological assessment and CD14/CD68-staining with subsequent analysis by flow cytometry. CD14-APC antibody was purchased from R\&D (R\&D Systems, Inc., Minneapolis, USA), CD68-FITC antibody was purchased from Dako (Dako Deutschland GmbH, Hamburg, Germany). Before adding MIF to the monocytes, we preincubated 20 $\mathrm{ng} / \mathrm{ml} \mathrm{MIF}$ and $2 \mu \mathrm{g} / \mathrm{ml}$ wild type Gremlin-1, mutant Gremlin-1, or control respectively, for $30 \mathrm{~min}$ at room temperature in RPMI medium supplemented with $10 \% \mathrm{FCS}$ and $1 \%$ penicillin/streptomycin $(100 \mu \mathrm{g} / \mathrm{ml}$ each). After preincubation of the MIF/Gremlin-1 protein mixtures, human monocytes were cultured in the presence of these preincubated proteins at $5 \% \mathrm{CO} 2$ and $37^{\circ} \mathrm{C}$ for 6 days.

\section{Statistical Analysis}

Data is presented as mean \pm standard error of mean (SEM). Statistical significance was determined using one-way ANOVA with Tukey HSD (honestly significant difference) post hoc test and unpaired twotailed Student's t test where needed. For statistical analysis Prism 4 and 6 software for windows (version 4.03 and version 6.07, GraphPad Software, Inc.) was used. A $p$-value $\leq 0.05$ was regarded as statistically significant.

\section{Results}

Generation of Gremlin-1 mutants and prediction of 3D-structure

In silico generated, mutated Gremlin-1 displays a significantly reduced binding to MIF in a computational docking simulation generated with Molegro Virtual Docker 5. The mutations are in the C-terminal region involving several positively charged amino acid residues (Fig. 1A). The estimated dissociation constant of mutant Gremlin-1 is $\mathrm{K}_{\mathrm{D}}=896 \pm 36 \mathrm{nM}$ compared to wild type Gremlin- 1 with $\mathrm{K}_{\mathrm{D}}=65 \pm 6 \mathrm{nM}$ and accordingly the predicted interaction energy between the mutated Gremlin-1 and MIF was higher than between wild type Gremlin-1 and MIF; precisely, mutated Gremlin-1/MIF -289.95 \pm 31.47 kJ/mol vs. wild type Gremlin-1/ MIF $-435.24 \pm 26.35 \mathrm{~kJ} / \mathrm{mol}$ (see table in Fig. 1A). The mutated Gremlin-1 clearly showed a different surface charge distribution than the wild type Gremlin-1.

Based on this estimated area which seems important for MIF binding, 4 mutant constructs based on the wild type Gremlin-1-Fc plasmid were obtained by site-directed mutagenesis PCR, namely the single amino acid exchange mutants K145A, K147A, R169A and R172A (numbering is based on the wild type Gremlin-1 protein (UniProt ID: 060565)). The alignment of the C-terminal amino acid sequences of the 4 mutants as well as that of wild type Gremlin-1 is shown in Fig. 1B. Since there is no NMR structure of Gremlin-1 currently available we performed in silico modelling of wild type Gremlin-1 and the mutant R172A with I-TASSER (Iterative Threading ASSEmbly Refinement) (Fig. 1B). This prediction suggests that the R172A-mutant could comprise a slightly altered three-dimensional folding compared to the wild type protein (Fig. 1B upper and middle panel) with a more bulky structure. The exact size and relative purity of the expressed wild type and mutant Gremlin-1 fusion proteins (schematic in Fig. 1C) was proved via SDS-PAGE and subsequent Western Blot (Fig. 1D).

\section{KARGER}




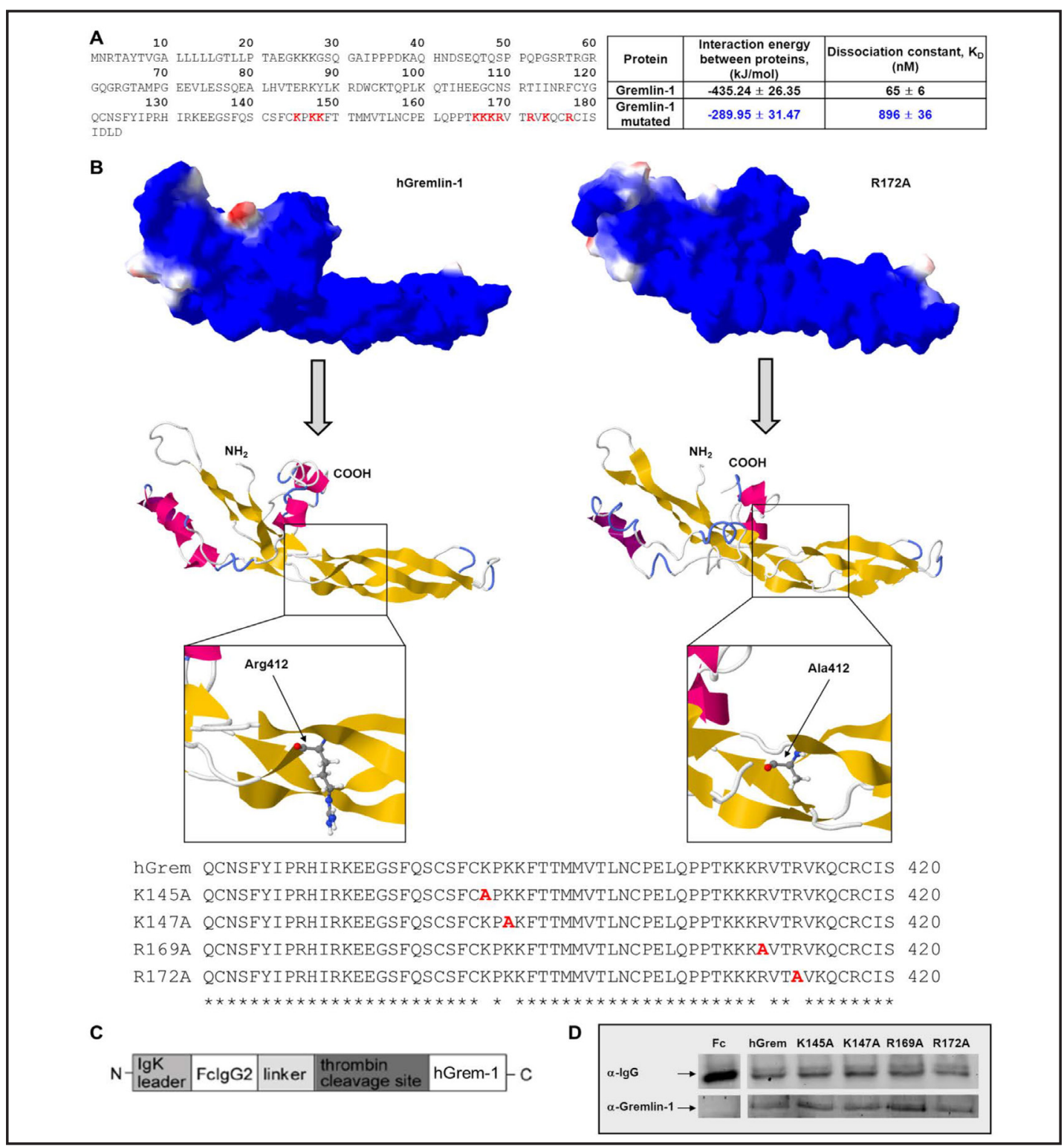

Fig. 1. Computer modelling and C-terminal sequence of Gremlin-1 mutants. (A) Computational modelling showing the predicted protein structure of murine wild type Gremlin-1 and mutated Gremlin-1. Murine and human Gremlin-1 share 97.83\% homology (98.75\% after shedding the signal peptide). Hypothetic mutations are shown in the sequence as red letters. The surface charge distribution is shown as follows: negative charge red, positive charge blue and neutral charge white. (B) Predicted three-dimensional structures of Gremlin-1 and R172A were obtained by LOMETS/I-TASSER. Predicted pdb-files were displayed using SwissPDBViewer (heat map of electrostatic surface potential, upper panel) and Jmol: an open-source Java viewer for chemical structures in 3D. http://www.jmol.org/ (middle panel). C-terminal sequences of wild type Gremlin-1 fusion protein, as well as those of the 4 single amino acid exchange mutants (lower panel, conserved amino acids are shown by asterisk *). (C) Schematic presentation of the fusion proteins, consisting of IgK (immunoglobulin kappa) leader, immunoglobulin G2 Fc (fragment crystallizable) tag, and the C-terminal Gremlin-1 part. (D) Western Blot analysis to prove the purity of the produced Gremlin-1 fusion proteins.

Loss-of-function mutant R172A fails to reduce MIF-induced monocyte migration

MIF-induced monocyte migration is a critical step in the development of atherogenic lesions $[5,6]$. Thus, we evaluated if our mutant Gremlin-1 proteins can inhibit MIF-induced 
A
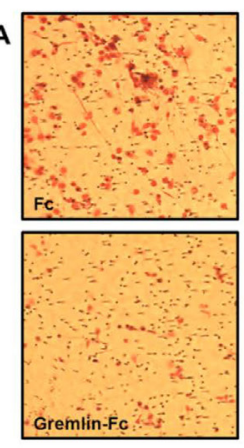

B

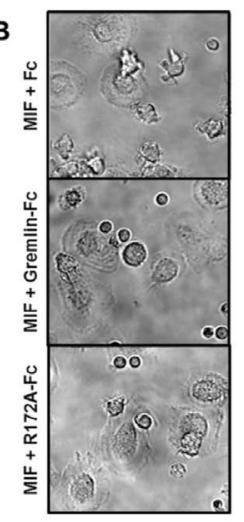

C

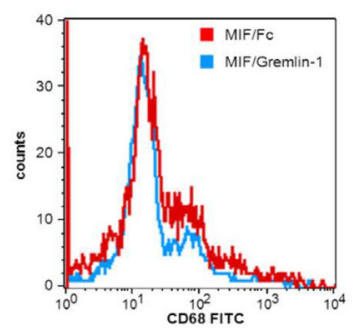

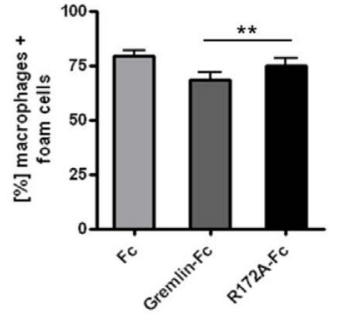

differentiation day 4
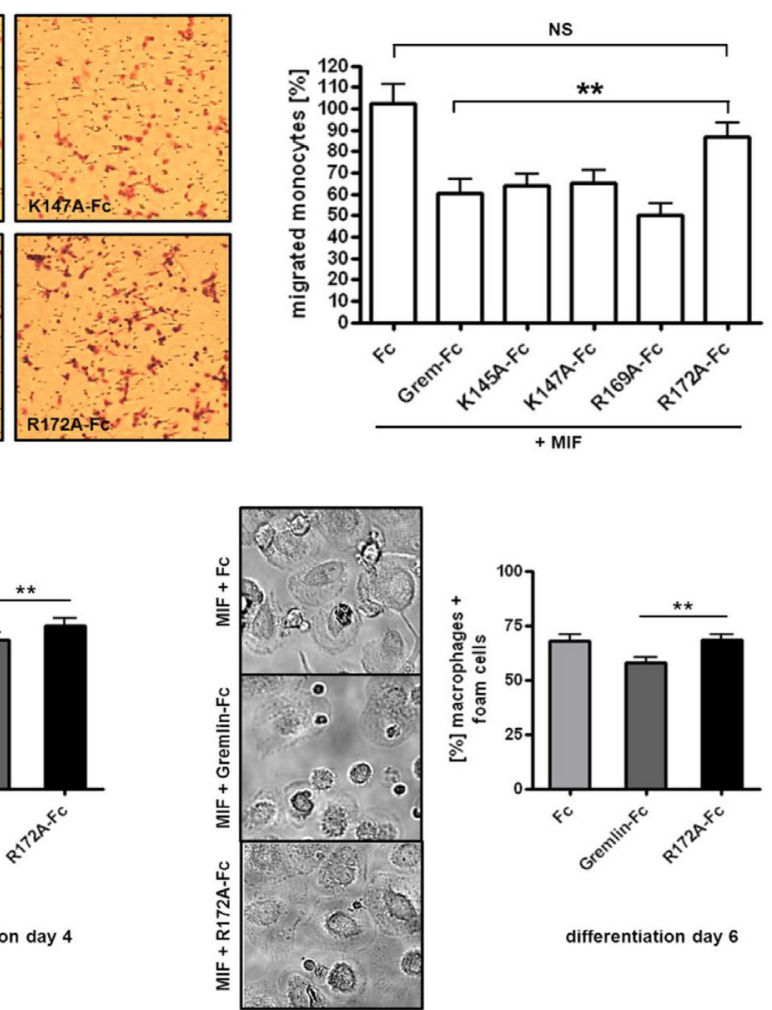

differentiation day 6

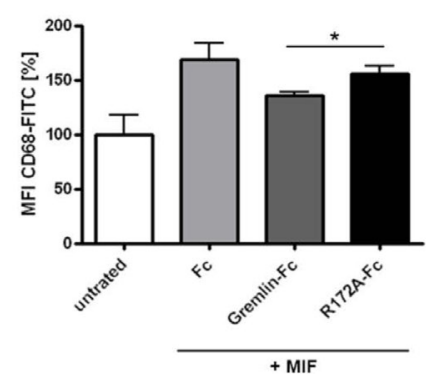

Fig. 2. Loss-of-function mutant R172A. (A) Migration of human monocytes. Representative pictures of the stained (May-Grünwald/Giemsa staining, left panel) membrane filter after 4 hours migration time. Quantification of 3 independent experiments evaluating monocyte migration towards MIF (20 ng/ml) with $30 \mathrm{~min}$ preincubation with $2 \mu \mathrm{g} / \mathrm{ml}$ fusion proteins (wild type Gremlin-1, or Gremlin-1 mutants K145A, K147A, R169A, or R172A), or control (Fc) respectively. NS not significant, ${ }^{*} p<0.05$. (B) Human monocytes were differentiated into macrophages/foam cells under the influence of MIF supplemented to the cell culture medium for 6 days. Prior (30 min) incubation of MIF and wild type/mutant Gremlin-1 was performed at room temperature. Cell numbers were counted on day 4 and 6 , and quantified in 3 independent experiments. $* p<0.05$. (C) Flow cytometric analyses of the percentage of CD68-positive cells in the total cell population after 6 days of MIF-induced differentiation. Prior incubation was performed as described above. On day 6 of macrophage/foam cell differentiation, cells were stained for CD68. Histogram overlays of MIF/Fc versus MIF/Gremlin-1-Fc, or MIF/R172A-Fc versus MIF/Gremlin-1-Fc, respectively, are shown in the left panel. Quantification from 3 independent experiments are shown in the right panel. ${ }^{*} p<0.05$.

monocyte migration. We preincubated wild type Gremlin-1-Fc, mutant proteins K145A, K147A, R169A, R172A and controls respectively with MIF for $30 \mathrm{~min}$, then allowed the monocytes to migrate towards the proteins for 4 hours. Wild type Gremlin- 1 significantly reduced MIF-induced monocyte migration, as previously shown [11] from $100 \%$ in the MIF/ 
Fc treated control cells to about $60 \%$ migrated monocytes $(60.58 \pm 6.40 \%, p<0.0001)$ as observed in the MIF/wild type Gremlin-1 treated populations. Three of the tested Gremlin-1 mutants reduced MIF-induced monocyte migration comparable to wild type Gremlin-1, in particular the K145A (64.09 $\pm 5.14 \%)$, K147A $(65.34 \pm 6.03 \%)$, and R169A (50.49 \pm $5.02 \%$ ) mutants, respectively (Fig. 2A). But one mutant, namely R172A, failed to reduce MIF-induced monocyte migration and interestingly showed significantly higher migration rates than Gremlin-1 treated populations (R172A/MIF $86.86 \pm 6.82 \%$ vs. Gremlin-1/MIF $60.58 \pm 6.40 \%, p=0.0413$ ). Preincubation of the Gremlin-1 mutant R172A with MIF showed no significant difference in the number of migrated monocytes as compared to the Fc/MIFpreincubated sets $(86.86 \pm 6.82 \%$ vs. $100 \%, p>0.05)$.

R172A does not inhibit MIF-induced monocyte differentiation into macrophages and foam cells

Monocyte differentiation into macrophages and foam cells plays an important role in atherosclerosis $[7,8,11,16]$. The MIF-induced monocyte differentiation into macrophages can be reduced by Gremlin-1 [11], but presently could not be reduced by the Gremlin-1 mutant R172A (Fig. 2B-C). Isolated human monocytes were differentiated into macrophages/foam cells over 6 days. Differentiation was induced by MIF (20 ng/ml) which was preincubated for $30 \mathrm{~min}$ with $2 \mu \mathrm{g} / \mathrm{ml}$ wild type Gremlin-1 or R172A fusion protein. During differentiation cell numbers were quantified on day 4 and day 6 (Fig. 2B). Only wild type Gremlin-1, but not the R172A fusion protein, could reduce the MIF-induced differentiation of monocytes into macrophages and foam cells. On day 4 Gremlin-1 significantly reduced MIF-induced macrophage/foam cell formation from about $90 \%$ to $80 \%$ (MIF/Fc $88.93 \pm 1.36 \%$ vs. MIF/ Gremlin-1 $81.78 \pm 1.18 \%, p=0.0003$ ), whereas mutant Gremlin-1 R172A was not able to reduce macrophage/foam cell differentiation and morphologically the cells looked like the Fc-treated cell populations (MIF/Fc $88.93 \pm 1.36 \%$ vs. R172A $86.50 \pm 1.08 \%, p>0.05$ ) (Fig. 2B, left panel). Differentiation into macrophages/foam cells was also significantly reduced by wild type Gremlin-1 (MIF/Fc $68.07 \pm 2.83 \%$ vs. MIF/Gremlin-1 $57.62 \pm 2.64 \%$, $p=0.0185$ ), on day 6 , and not by mutant Gremlin-1 R172A (MIF/Fc $68.07 \pm 2.83 \%$ vs. $68.27 \pm 2.58 \%, p>0.05$ ) (Fig. $2 \mathrm{~B}$, right panel). Mutant Gremlin-1 R172A was significantly less active in preventing MIF-induced monocyte differentiation, in particular MIF/R172A induced $86.50 \pm 1.08 \%$ (day 4 ), or $68.27 \pm 2.58 \%$ (day 6) macrophage/foam cell formation, compared to MIF/wild type Gremlin-1, which induced only $81.78 \pm 1.18 \%$ (day 4), or 57.62 $\pm 2.64 \%$ (day 6) with a $p$-value of 0.0209 (day 4), or 0.0160 (day 6), respectively. On day 6 macrophage/foam cell development was analyzed by flow cytometry. We observed that consistent with the number of cells counted by morphologic features on day 6 (Fig. 2B), MIF/wild type Gremlin-1-treated cells showed significantly less CD68-positive cells (136.0 $\pm 3.02 \%$ ), compared to the MIF/Fc-treated population, which showed $168.2 \pm 15.98 \%$ CD68-positive cells (Fig. 2C). The Gremlin-1 mutant R172A on the other hand showed no significant reduction in CD68-positive cells, MIF/R172A-treated culture sets showed levels of CD68-positive cells comparable to the MIF/Fc-treated populations (MIF/R172 $155.7 \pm$ $6.81 \%$ vs. MIF/Fc $168.2 \pm 15.98 \%, p>0.05$ ).

\section{Discussion}

MIF-dependent monocyte infiltration and macrophage formation are pivotal steps in atherosclerosis, plaque vulnerability and vascular inflammation [5]. Gremlin-1 reduces macrophage differentiation by antagonizing MIF, a cytokine critically involved in atherosclerotic plaque progression and vulnerability. Gremlin-1 binds to MIF with high affinity $\left(K_{D}=54 \mathrm{~nm}\right)$, as proven by surface plasmon resonance analysis and coimmunoprecipitation, and reduces MIF-induced release of TNF- $\alpha$ from macrophages. Thus, Gremlin-1 acts as an endogenous antagonist of MIF and this defines a role for Gremlin-1/ MIF interaction in the context of atherosclerosis. 
Previously, we demonstrated that recombinant fusion molecule, ${ }_{\mathrm{m}}^{\text {Gremlin-1-Fc, }}$ binds with high affinity $\left(K_{D}=76 \mathrm{~nm}\right)$ to MIF and substantially reduces the cellularity of atherosclerotic lesions in $A p o E^{-/-}$mice, most prominently due to a decrease in macrophage infiltration [8]. In the present study we used in silico computer modelling to further characterize the binding activity of Gremlin-1 to the globular MIF protein. The computerbased analysis postulated that the C-terminal end of Gremlin- 1 is critical for binding to MIF. In silico generated mutations of positively charged amino acid residues (arginine and lysine) within the C-terminus of Gremlin-1 substantially reduced the estimated binding affinity of Gremlin-1 for MIF in a computational docking prediction.

To further analyze the molecular mechanisms of Gremlin-1/MIF interaction we constructed various recombinant Gremlin- 1 fusion proteins using site directed mutagenesis approach. We generated single amino acid exchange mutants of two C-terminal lysine residues (K145A and K147A), as well as of two arginine residues (R169A and R172A). We found that R172A, where the positively charged arginine residue was mutated to an uncharged alanine residue, showed a loss of activity to antagonize MIF-induced monocyte function. R172A could not attenuate MIF-triggered monocyte migration as validated using a modified Boyden-chamber migration assay. Interestingly we could also show that exchange of a nearby arginine residue (Arg169) with alanine showed no reduction of MIF-inhibiting function at all, indeed this R169A mutant rather showed a tendency to be even more active than wild type Gremlin-1 in the monocyte migration assay. Further, we analyzed the effect of this loss-of-function mutant in a monocyte differentiation assay as described previously $[8,16]$. As documented previously, wild type Gremlin-1 but not the R172A mutant inhibited MIF-induced monocyte-derived macrophage/foam cell differentiation. This data suggests that the C-terminus of Gremlin-1 is critically involved in counteracting MIF.

In conclusion, our present data provides evidence that the C-terminus of Gremlin-1 plays an important role in regulation of MIF-induced monocyte functions and offers a novel strategy for development of pharmacological approaches to target MIF in various diseases including vascular inflammation and atherogenesis.

\section{Acknowledgements}

This project was supported by the Deutsche Forschungsgemeinschaft (Klinische Forschungsgruppe-KFO-274: 'Platelets - Molecular Mechanisms and Translational Implications').

\section{Disclosure Statement}

The authors declare that there are no conflicts of interest.

\section{References}

1 Topol LZ, Bardot B, Zhang Q, Resau J, Huillard E, Marx M, Calothy G, Blair DG: Biosynthesis, post-translation modification, and functional characterization of Drm/Gremlin. J Biol Chem 2000;275:8785-8793.

2 Michos 0, Goncalves A, Lopez-Rios J, Tiecke E, Naillat F, Beier K, Galli A, Vainio S, Zeller R: Reduction of BMP4 activity by gremlin 1 enables ureteric bud outgrowth and GDNF/WNT11 feedback signalling during kidney branching morphogenesis. Development 2007;134:2397-2405.

3 Shi W, Zhao J, Anderson KD, Warburton D: Gremlin negatively modulates BMP-4 induction of embryonic mouse lung branching morphogenesis. Am J Physiol Lung Cell Mol Physiol 2001;280:L1030-1039.

4 Khokha MK, Hsu D, Brunet LJ, Dionne MS, Harland RM: Gremlin is the BMP antagonist required for maintenance of Shh and Fgf signals during limb patterning. Nat Genet 2003;34:303-307. 


\section{Cellular Physiology Cell Physiol Biochem 2016;38:801-808 \begin{tabular}{ll|l} 
DOI: 10.1159/000443035 & $\begin{array}{l}\text { O 2016 The Author(s). Published by S. Karger AG, Basel } \\
\text { www.karger.com/cpb }\end{array}$ \\
and Biochemistry Published online: February 15, 2010 &
\end{tabular} \\ Beck et al.: Gremlin-1 Mutants}

5 Bernhagen J, Krohn R, Lue H, Gregory JL, Zernecke A, Koenen RR, Dewor M, Georgiev I, Schober A, Leng L, Kooistra T, Fingerle-Rowson G, Ghezzi P, Kleemann R, McColl SR, Bucala R, Hickey MJ, Weber C: MIF is a noncognate ligand of CXC chemokine receptors in inflammatory and atherogenic cell recruitment. Nat Med 2007;13:587-596.

6 Weber C, Kraemer S, Drechsler M, Lue H, Koenen RR, Kapurniotu A, Zernecke A, Bernhagen J: Structural determinants of MIF functions in CXCR2-mediated inflammatory and atherogenic leukocyte recruitment. Proc Natl Acad Sci USA 2008;105:16278-16283.

7 Libby P, Ridker PM, Hansson GK: Progress and challenges in translating the biology of atherosclerosis. Nature 2001;473:317-325.

8 Muller II, Schonberger T, Schneider M, Borst O, Ziegler M, Seizer P, Leder C, Muller KA, Lang M, Appenzeller F, Lunov O, Buchele B, Fahrleitner M, Olbrich M, Langer H, Geisler T, Lang F, Chatterjee M, de Boer JF, Tietge UJ, Bernhagen J, Simmet T, Gawaz M: Gremlin-1 is an inhibitor of macrophage migration inhibitory factor and attenuates atherosclerotic plaque growth in ApoE-/- Mice. J Biol Chem 2013;288:31635-31645.

9 Muller II, Muller KA, Karathanos A, Schonleber H, Rath D, Vogel S, Chatterjee M, Schmid M, Haas M, Seizer P, Langer H, Schaeffeler E, Schwab M, Gawaz M, Geisler T: Impact of counterbalance between macrophage migration inhibitory factor and its inhibitor Gremlin-1 in patients with coronary artery disease. Atherosclerosis 2014;237:426-432.

10 Schmidt R, Bultmann A, Ungerer M, Joghetaei N, Bulbul O, Thieme S, Chavakis T, Toole BP, Gawaz M, Schomig A, May AE: Extracellular matrix metalloproteinase inducer regulates matrix metalloproteinase activity in cardiovascular cells: implications in acute myocardial infarction. Circulation 2006;113:834-841.

11 Muller II, Chatterjee M, Schneider M, Borst O, Seizer P, Schonberger T, Vogel S, Muller KA, Geisler T, Lang F, Langer H, Gawaz M: Gremlin-1 inhibits macrophage migration inhibitory factor-dependent monocyte function and survival. Int J Cardiol 2014;176:923-929.

12 Ziegler M, Elvers M, Baumer Y, Leder C, Ochmann C, Schonberger T, Jurgens T, Geisler T, Schlosshauer B, Lunov O, Engelhardt S, Simmet T, Gawaz M: The bispecific SDF1-GPVI fusion protein preserves myocardial function after transient ischemia in mice. Circulation 2012;125:685-696.

13 Baumer Y, Leder C, Ziegler M, Schonberger T, Ochmann C, Perk A, Degen H, Schmid-Horch B, Elvers M, Munch G, Ungerer M, Schlosshauer B, Gawaz M: The recombinant bifunctional protein alphaCD133-GPVI promotes repair of the infarcted myocardium in mice. J Thromb Haemost 2012;10:1152-1164.

14 Ross R: Atherosclerosis--an inflammatory disease. N Engl J Med 1999;340:115-126.

15 Boers W, Aarrass S, Linthorst C, Pinzani M, Elferink RO, Bosma P: Transcriptional profiling reveals novel markers of liver fibrogenesis: gremlin and insulin-like growth factor-binding proteins. J Biol Chem 2006;281:16289-16295.

16 Daub K, Langer H, Seizer'P, Stellos K, May AE, Goyal P, Bigalke B, Schonberger T, Geisler T, Siegel-Axel D, Oostendorp RA, Lindemann S, Gawaz M: Platelets induce differentiation of human CD34+ progenitor cells into foam cells and endothelial cells. FASEB J 2006;20:2559-2561. 